Chirurg 2021 · 92:497-498

https://doi.org/10.1007/s00104-021-01395-9

Angenommen: 15. März 2021

Online publiziert: 26. April 2021

(c) Springer Medizin Verlag GmbH, ein Teil von

Springer Nature 2021

\section{Redaktion}

I. Gockel, Leipzig

Das Magenkarzinom ist weltweit eine führende Todesursache: Es ist die dritthäufigste Ursache der krebsbedingten Todesfälle nach dem Lungen- und kolorektalen Karzinom. Zudem stellt es die am fünfthäufigste diagnostizierte Krebsart dar. Die höchsten Inzidenzraten finden sich in Ostasien, Osteuropa und Südamerika. Seit Jahrzehnten ist in Deutschland - wie auch in anderen Industrienationen - ein stetiger Rückgang der Erkrankungs- und Sterberaten beim Magenkarzinom zu beobachten. Während die Inzidenz von Karzinomen des mittleren und unteren Magendrittels in der westlichen Hemisphäre sinkt, nimmt sie für Adenokarzinome im proximalen Magendrittel und am ösophagogastralen Übergang (AEG-Tumoren) $\mathrm{zu}$ [1]. Die anatomische Lokalisation des Tumorursprungs korreliert mit vorherrschenden molekularen Charakteristika der Erkrankung [2]. Im proximalen Magen entstehen überwiegend Karzinome mit chromosomaler Instabilität (CIN). Neben den häufigen p53-Mutationen treten beim CIN-Magenkarzinom auch Amplifikationen von Rezeptortyrosinkinasen wie humaner epidermaler Wachstumsfaktor 2 (HER2) auf. Der deutlich seltenere Epstein-Barr-Virus (EBV)-assoziierte Subtyp ist eher im Magenfundus- und -korpusbereich $\mathrm{zu}$ finden; der hypermutierte mikrosatelliteninstabile (MSI-)Subtyp in den distalen Magenabschnitten. Genomisch stabile (GS-)Karzinome werden in allen Magenabschnitten gefunden, weisen häufig einen diskohäsiven Wachstumstyp (diffuser Typ nach Laurén) und Alterationen von Adhäsionsproteinen wie Cadherin, RhoA oder Claudin-18 auf [2]. Aufgrund

\title{
Ines Gockel
}

Klinik und Poliklinik für Viszeral-, Transplantations-, Thorax- und Gefäßchirurgie, Department für Operative Medizin (DOPM), Universitätsklinikum Leipzig, Leipzig, Deutschland

\section{Update Magenkarzinom}

der biologischen Heterogenität sowie des aggressiven Wachstums- und Metastasierungsverhaltens stellt die Behandlung von Patienten mit Magenkarzinomen große Anforderungen an alle beteiligten medizinischen Disziplinen.

Mit Ausnahme klar definierter Subgruppen der Frühkarzinome, die einer endoskopischen Resektion (ER) unterzogen werden können, ist die einzige kurative Therapie bis heute die onkologische Magenresektion (totale/subtotale oder transhiatal erweiterte Gastrektomie) mit systematischer Lymphknotendissektion [3].

\section{\) Das oligometastasierte Magenkarzinom ist eine besondere, interdisziplinäre Herausforderung}

Trotz leitliniengerechter Chirurgie ist die Prognose oftmals schlecht, da das Magenkarzinom dazu tendiert, bereits in frühen Stadien zu metastasieren (lymphogen, hämatogen, peritoneal oder als sog. „Abtropfmetastasen“, z. B. im Sinne eines Krukenberg-Tumors). Fundierte Einblicke in die heterogenen immunhistopathologischen Charakteristika sind notwendig, um personalisierte Therapien für spezielle Patientenpopulationen zu entwickeln. Als molekulare Zielstruktur hat sich HER2 etabliert. Neue HER2-gerichtete Substanzen könnten in Zukunft die Therapielandschaft des HER2-positiven Magenkarzinoms ergänzen. Immuntherapie ist wirksam bei chemotherapierefraktären und bei MSI-Magenkarzinomen, steht in Deutschland jedoch noch nicht zur Behandlung außerhalb von Studien zur Verfügung. Nivolumab plus Ipilimumab wird jetzt als adjuvante Therapie in kurativer Intention bei unbefriedigendem Ansprechen auf eine präoperative Chemotherapie untersucht.

So steht die neoadjuvante und perioperative Therapie des Magenkarzinoms, insbesondere bezugnehmend auf aktuelle Studien und neue Biomarker, im Fokus des Beitrags von Stocker et al. Zudem wird ein Ausblick auf aktuelle Entwicklungen auf dem Gebiet der medikamentösen perioperativen Therapie gegeben. Die Magenkarzinomchirurgie hat in den letzten 20 Jahren einen deutlichen Wandel erfahren. Weber und Mitautoren stellen ein evidenzbasiertes Update der verschiedenen Rekonstruktionsverfahren nach subtotaler Magenresektion und Gastrektomie dar. Die Therapie des oligometastasierten Magenkarzinoms stellt eine besondere, interdisziplinäre Herausforderung dar. Aufgrund der Resultate der deutschen AIO-FLOT-3-Studie, in der sich das mediane Überleben oligometastasierter Patienten mit multimodaler Therapie verdoppelte, wurde die AIO-FLOT-5(RENAISSANCE)-Studie initiiert. Jung et al. bieten eine Übersicht über die Therapieoptionen und aktuelle Studien dieser besonderen Situation der Oligometastasierung. Den Stellenwert der zytoreduktiven Chirurgie und hyperthermen intraperitonealen Chemotherapie (HIPEC) beim peritoneal metastasierten Magenkarzinom beleuchten Gronau und Mitautoren. Abschließend wird im Artikel von Berlth et al. auf Indikation und Stellenwert der minimal-invasiven Gastrektomie sowie aktuelle technische Weiterentwicklungen 
eingegangen. Hier stehen die Robotik und das intraoperative Imaging beim Magenkarzinom im Vordergrund.

Ich gehe fest davon aus, dass das Themenschwerpunktheft „Update Magenkarzinom" fundierte Einblicke in aktuelle Therapiemöglichkeiten mit innovativen Aspekten von klinischer Relevanz bietet.

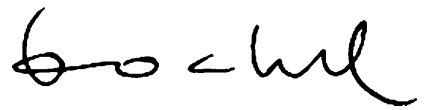

Univ.-Prof. Dr. Ines Gockel, MBA

\section{Korrespondenzadresse}

\section{Univ.-Prof. Dr. Ines Gockel, MBA}

Klinik und Poliklinik für ViszeralTransplantations-, Thorax- und Gefäßchirurgie, Department für Operative Medizin (DOPM), Universitätsklinikum Leipzig Liebigstr. 20, 04103 Leipzig, Deutschland ines.gockel@medizin.uni-leipzig.de

Interessenkonflikt. I. Gockel gibt an, dass kein Interessenkonflikt besteht.

\section{Literatur}

1. Bray F, Ferlay J, Soerjomataram I, Siegel RL, Torre LA, Jemal A (2018) Global cancer statistics 2018: GLOBOCAN estimates of incidence and mortality worldwide for 36 cancers in 185 countries. CA Cancer JClin 68(6):394-424

2. Cancer Genome Atlas Research Network (2014) Comprehensive molecular characterization of gastric adenocarcinoma. Nature 513(7517:202-209

3. MoehlerMetal (2019) S3-Leitlinie Magenkarzinom - Diagnostik und Therapie der Adenokarzinome des Magens und des ösophagogastralen Übergangs - Langversion 2.0 - August 2019. AWMFRegisternummer: 032/009OL. Z Gastroenterol 57(12):1517-1632

\section{Hackert, Thilo, Croner, Roland S. (Hrsg.) \\ Roboterassistierte Viszeral- und Thoraxchirurgie}

Berlin Heidelberg: Springer-Verlag 2021, 1. Auflage, 160 S., 180 Abb., (ISBN: 978-3662-60456-4), Hardcover 79,99 EUR

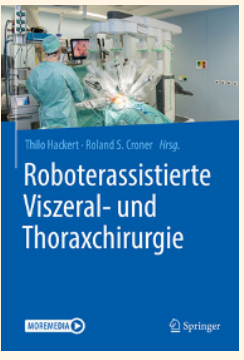

Roboterassistierte Operationen nehmen in Zahl und Komplexität kontinuierlich zu, damit geht auch ein Trend zur flächendeckenden Anwendung dieser Operationstechniken einher. Im Bereich der minimalinvasiven Chirurgie ist im Laufe der nächsten Jahre sicherlich von einer relativen Zunahme von roboterassistierten Operationen und Reduktion der "klassisch" laparoskopischen Operationen auszugehen. Somit ist es sinnvoll, die von Experten bereits gemachten Erfahrungen strukturiert zu sammeln und den Anwendern dieser neuen Techniken zur Verfügung zu stellen.

Im Buch „Roboterassistierte Viszeral- und Thoraxchirurgie" von Thilo Hackert und Roland S. Croner ist eine exzellente strukturierte Darstellung dieser Thematik gelungen. Das Buch beginnt mit einem umfassenden Kapitel zum Thema "Training für die roboterassistierte Chirurgie". Hierbei wird dieser wichtige Bereich sehr strukturiert dargestellt und hilft bei der Planung zur Ausbildung einzelner Roboterchirurgen und auch zur Etablierung eines umfassenden Ausbildungscurriculums. In den weiteren Kapiteln umfasst dieses Buch weitgehend alle operationsrelevanten Aspekte und beleuchtet die einzelnen Organbereiche im Detail. Die einzelnen Operationen sind hinsichtlich Operations-Setup, intraoperativer Herangehensweise, Trouble Shooting und Evidenz aufgearbeitet und mit Hilfe von Schaubildern und intraoperativer Fotodokumentation unterstützt. Zusätzlich sind in das Buch sehr gute Videosequenzen zu einzelnen Operationsschritten integriert. Ein wichtiger Aspekt bei der Bewertung einer neuen Operationstechnik ist der kritische Umgang mit der Neuheit. Hackert und Croner lassen es nicht vermissen, auch kritisch die Nachteile der Roboterassistierten Chirurgie darzustellen.
Zusammenfassend ist zu sagen, dass das Buch „Roboterassistierte Viszeral- und Thoraxchirurgie" eine qualitativ hochwertige Zusammenstellung der relevanten operationsspezifischen Aspekte dieser neuen Technik ist und sicherlich zur Weiterentwicklung der Roboterassistierten Chirurgie beitragen kann.

S. Fichtner-Feigl (Freiburg) 